Pesq. Vet. Bras. 30(6):471-478, junho 2010

\title{
Determinação da taxa metabólica basal em cutias, Dasyprocta azarae, por calorimetria indireta ${ }^{1}$
}

\author{
Harald F.V. Brito ${ }^{2 *}$, Rogério R. Lange ${ }^{3}$, José R. Pachaly ${ }^{4}$ e Ivan Deconto ${ }^{5}$
}

\begin{abstract}
Brito H.F.V., Deconto I., Lange R.R. \& Pachaly J.R. 2010. [Determination of the basal metabolic rate in agoutis, Dasyprocta azarae, by indirect calorimetry.] Determinação da taxa metabólica basal em cutias, Dasyprocta azarae, por calorimetria indireta. Pesquisa Veterinária Brasileira 30(6):471-478. Instituto Qualittas de Pós-Graduação, Rua Conde D'Eu 38, Campinas, SP 13100-009, Brazil. E-mail: haraldvet@yahoo.com.br

The best way to compare different organisms is the basal metabolic rate, a fundamental interrelation existent among all living beings. Direct measures of oxygen and carbon dioxide concentrations by evaluation of inspired and expired air can be used to measure metabolic rate. So, this research was done in order to measure basal and specific metabolic rates in agoutis (Dasyprocta azarae), and reexamine the scaling of basal metabolism in this species. There were used 34 adult healthy agoutis ( 9 non-castrated males, 9 castrated males, and 16 females), that belong to the wild animal scientific breeding facility of the Natural History Museum of the Curitiba city, State of Paraná, Brazil. After a six-hour fasting the animals were placed in special boxes under controlled temperature $\left(22.0 \pm 1.0^{\circ} \mathrm{C}\right)$, and submitted to measuring of the basal metabolic rate, by indirect calorimetry. It was used the Deltatrac®II metabolic monitor, usually indicated to measure carbon dioxide production $\left(\mathrm{VCO}_{2}\right)$ and oxygen consumption $\left(\mathrm{VO}_{2}\right)$ in human beings, by measuring variations in the concentration of $\mathrm{VCO}_{2}$ and of $\mathrm{VO}_{2}$, with a precision of $0.01 \%$. The specific metabolic rate was calculated after determination of the basal metabolic rate and the obtained data were analyzed by inductive statistics. The hypotheses tests for comparison among samples indicated that the specific metabolic rate is higher in non-castrated males than in females and castrated males (significance of $5 \%$ ), and that the specific metabolic rate of females and castrated males are equivalent (significance of $1 \%$ ). In addition, analysis of the correlation of experimental points indicates that another variable beyond body size affects the metabolic rate of non-castrated males (significance of 1\%), and therefore new studies on the metabolism of Dasyprocta azarae are required.
\end{abstract}

INDEX TERMS: Agouti, Dasyprocta azarae, basal metabolic rate, indirect calorimetry.

RESUMO.- A melhor ferramenta para comparação fisiológica entre organismos diferentes é a taxa metabólica basal, interrelação fundamental que existe entre todos os seres vivos.

\footnotetext{
1 Recebido em 19 de setembro de 2010.

Aceito para publicação em 2 de março de 2010

2 Instituto Qualittas de Pós-Graduação (IQPG), Rua Conde D'Eu 38, Campinas, SP 13100-009, Brasil. *Autor para correspondência: haraldvet@yahoo.com.br

${ }^{3}$ Curso de Medicina Veterinária, Universidade Paranaense (Unipar), Praça Mascarenhas de Moraes s/n, Umuarama, PR 87502-210, Brasil. E-mail: pachaly@uol.com.br

${ }^{4}$ Curso de Medicina Veterinária, UFPR, Curitiba, PR. E-mail: rrlange@ufpr.br

5 Curso de Medicina Veterinária, Universidade Federal do Paraná (UFPR), Rua dos Funcionários s/n, Curitiba, PR 80.035-050, Brasil. Email: deconto@ufpr.br
}

Mensurações diretas das concentrações de oxigênio e dióxido de carbono, pela análise do ar inspirado e expirado, podem ser usadas para a mensuração de taxa metabólica. Este trabalho foi executado com o propósito de aferir as taxas metabólicas basal e específica, e reexaminar o escalonamento do metabolismo basal em cutias (Dasyprocta azarae). Foram utilizadas 34 cutias (D. azarae) adultas sadias, sendo 9 machos não castrados, 9 machos castrados e 16 fêmeas, pertencentes ao plantel do Criadouro Científico do Museu de História Natural Capão da Imbuia, Curitiba, PR. Os animais passaram por jejum prévio de 6 horas e foram acondicionados em caixas especiais, com temperatura ambiente controlada $\left(22,0 \pm 1,0^{\circ} \mathrm{C}\right)$, sendo então submetidos à aferição da taxa metabólica basal, por calorimetria indireta. Empregou-se o monitor metabólico Deltatrac ${ }^{\circledR}$ II, (Datex- 
Ohmeda, Finlândia) usualmente indicado para a mensuração da produção de dióxido de carbono $\left(\mathrm{VCO}_{2}\right)$ e do consumo de oxigênio $\left(\mathrm{VO}_{2}\right)$ em seres humanos, por meio da mensuração das variações na concentração de $\mathrm{VCO}_{2}$ e de $\mathrm{VO}_{2}$, com uma precisão de $0,01 \%$. Após a aferição da taxa metabólica basal, foi calculada a taxa metabólica específica, e efetuada a análise dos dados por estatística indutiva. Os testes de hipóteses para comparação entre amostras indicaram que a taxa metabólica específica de machos não castrados é maior que a de fêmeas e machos castrados ( $5 \%$ de significância), e que a taxa metabólica específica de fêmeas e machos castrados é equivalente ( $1 \%$ de significância). Constatou-se ainda, com a análise da correlação de pontos experimentais, que outra variável que não o tamanho corporal afeta a taxa metabólica dos machos não castrados (1\% de significância), o que indica a necessidade de novos estudos sobre o metabolismo de Dasyprocta azarae, sugerindo-se a realização de aferição da taxa metabólica basal e aferição simultânea da concentração sérica de testosterona, estradiol e cortisol para os três grupos.

TERMOS DE INDEXAÇÃO: Cutia, Dasyprocta azarae, taxa metabólica basal, calorimetria indireta.

\section{INTRODUÇÃO}

O termo metabolismo significa literalmente "troca" (Ganong 1989) e é usado para indicar todas as transformações químicas e energéticas que ocorrem no organismo de um ser vivo (Ganong 1989, Guyton \& Hall 2002). Normalmente é expresso em termos da velocidade de liberação de calor durante as reações químicas (Guyton \& Hall 2002), denominada taxa metabólica (Ganong 1989). Invariavelmente, os valores das taxas metabólicas mensuradas se situam numa faixa entre um valor mínimo, denominado taxa metabólica basal, e um valor superior, denominado taxa metabólica máxima (Withers 1992).

Taxa metabólica basal é o valor medido quando um animal endotérmico se encontra quieto, inativo, não digerindo qualquer alimento, sem sofrer qualquer tipo de estresse e mantido sob temperatura ambiental ótima (Withers 1992), e pode ser calculada por meio da determinação do consumo de oxigênio nestas condições (Ganong 1989, Guyton \& Hall 2002). Dividindo-se a taxa metabólica basal pela massa corporal do animal, obtém-se o valor denominado taxa metabólica específica (TME), definida como a menor taxa metabólica por unidade de massa (Pachaly \& Brito 2000, 2001, Pachaly 2006).

O tamanho corporal é a característica isolada mais importante de um organismo, influenciando caracteres fundamentais, como o dispêndio energético para manutenção, porém, as relações existentes entre estes caracteres e o tamanho corporal são altamente variáveis. Alguns caracteres diminuem com o aumento do tamanho, e outros podem ser diretamente proporcionais à massa corporal. A maioria, entretanto, tem relação positiva com a massa corporal elevada a uma potência menor que uma unidade, o que significa simplesmente que a mudança em um caractere é proporcionalmente menor que a mudança no tamanho (McNab 1988).
Um dos problemas mais intrigantes e ainda não resolvidos na fisiologia animal comparativa é observado nessa relação entre a taxa metabólica e a massa corporal. Um elefante é maior que um camundongo, e tem a taxa metabólica total maior, mas a questão fundamental diz respeito à regra de proporcionalidade (Withers 1992). A taxa metabólica de animais não é diretamente proporcional à massa corporal, porém relaciona-se à massa na forma geral de uma curva de potência, representada pela equação $\boldsymbol{y}=\boldsymbol{a} \cdot \boldsymbol{M}^{\boldsymbol{b}}$, onde $\boldsymbol{a}$ representando a taxa metabólica quando $\boldsymbol{M}=1$, é a intercepção, e bé o expoente de massa, ou inclinação para a equação logaritmicamente transformada (Withers 1992).

A expressão gráfica de $\boldsymbol{y}$ contra $\boldsymbol{M}$ deve então seguir uma linha reta, com inclinação $\boldsymbol{b}$ e intercepção $\boldsymbol{a}$ (Feldman \& McMahon 1983, Withers 1992). Os valores de $\boldsymbol{a}$ e $\boldsymbol{b}$ podem ser estimados graficamente, e a análise de regressão linear não apenas revela uma linha de quadrados mínimos e estima numericamente a inclinação e a intercepção, como também os desvios-padrão de estimativa, que indicam quão precisamente aqueles parâmetros são estimados pelos dados (Feldman \& McMahon 1983). Kleiber empregou esta abordagem em seu estudo clássico sobre o metabolismo basal, observando que a inclinação de regressão do expoente de massa b era próxima a $3 / 4$, ou 0,75 (Feldman \& McMahon 1983, Withers 1992), demonstrando com isso que a taxa metabólica de mamíferos e aves de grande porte é proporcionalmente menor que a esperada por proporcionalidade direta, e que a taxa metabólica de aves e mamíferos de pequeno porte é maior que a esperada pela mesma proporcionalidade (Withers 1992). Segundo Pachaly \& Brito $(2000,2001)$ e Pachaly $(2006)$ o expoente 0,75 tem sido utilizado para cálculo de taxa metabólica basal de vertebrados, com diversos propósitos, tanto em pesquisa básica quanto aplicada.

A fórmula geral para o cálculo da taxa metabólica basal dos animais vertebrados, tanto domésticos como selvagens, segundo Gibbons et al. (1988), Sedgwick (1988a,b), Sedgwick \& Pokras (1988), Sedgwick et al. (1990), Mader (1991), Sedgwick (1991) Fowler (1993), Jacobson (1995), Dorrestein (1997), Gamble etal. (1997), Heard (1997), Pachaly \& Brito (2000, 2001) e Pachaly (2006), é expressa pela equação TMB $=\boldsymbol{a} \cdot \boldsymbol{M}^{\mathbf{0 , 7 5}}$.

Como mais de $95 \%$ da energia consumida no organismo provém de reações do oxigênio com os alimentos (Guyton \& Hall 2002), a quantidade de oxigênio usada nos processos de oxidação pode ser usada para determinação da taxa metabólica (Schmidt-Nielsen 1997, Guyton \& Hall 2002), pois a quantidade de calor produzido para cada litro de oxigênio usado no metabolismo permanece quase constante, independente de gordura, carboidrato ou proteína serem oxidados. Quando um litro de oxigênio é metabolizado com carboidrato, ocorre liberação de aproximadamente 5,0 quilocalorias de energia; quando metabolizado com gordura, são liberadas 4,7 quilocalorias, e com proteína, liberam-se 4,5 quilocalorias (Schmidt-Nielsen 1997, Guyton \& Hall 2002). Uma vez que a diferença entre o valor mais alto e o mais baixo é de apenas $10 \%$, é habitual usar o valor médio de 4,8 quilocalorias por litro de oxigênio con- 
sumido, como medida da taxa metabólica (Schmidt-Nielsen 1997), sendo este valor denominado de equivalente energético do oxigênio (Schmidt-Nielsen 1997, Withers 1992). Ao utilizar este equivalente energético, é possível calcular, com elevado grau de precisão, a velocidade de liberação de calorias pelo organismo, a partir da quantidade utilizada em determinado período de tempo (Guyton \& Hall 2002). O maior erro resultante do uso desse número médio é $6 \%$, mas se o combustível utilizado for uma mistura dos alimentos comuns (carboidrato, gordura e proteína), em geral o erro será insignificante (Schmidt-Nielsen 1997). O erro pode ser menor que 3\% ( $<3 \%)$, quando a excreção de nitrogênio urinária não é medida, e se assume que o quociente respiratório (QR) é 0,8 ; independentemente da proteína, gordura e mistura de carboidratos que estão sendo catabolizados por carnívoros, onívoros ou herbívoros. E pode ser ainda menor em quase todos os casos em que 0 QR é medido e utilizado para derivar fatores de conversão de energia (Gessaman \& Nagy 1988).

Segundo Withers (1992), mensurações do metabolismo pela determinação do consumo de oxigênio $\left(\mathrm{VO}_{2}\right)$ e produção de dióxido de carbono $\left(\mathrm{VCO}_{2}\right)$ não são diretamente equivalentes à taxa de produção de calor, mas podem ser relacionadas com a produção de calor através da estequiometria da reação química. Consequentemente, tais técnicas de mensuração da taxa metabólica são também denominadas calorimetria indireta.

Mensurações diretas das concentrações de oxigênio e dióxido de carbono pela análise do ar inspirado e expirado, ao invés da variação no volume, são também comumente usadas para a mensuração da taxa metabólica (Withers 1992).

Segundo Sayeed (2003), o monitor metabólico Deltatrac-Il é um sistema não invasivo, indicado para fazer esta mensuração de produção de dióxido de carbono $\left(\mathrm{VCO}_{2}\right)$ e consumo de oxigênio $\left(\mathrm{VO}_{2}\right)$. A concentração de dióxido de carbono e oxigênio do ar que entra na caixa onde o animal está acondicionado é mensurada pelo monitor após a captação de amostras deste ar por uma linha externa ligada à entrada da caixa. O equipamento pode ser regulado para retirar o ar da caixa por meio de um ventilador centrífugo, a uma taxa constante de 3,1 ou 10,1 litros por minuto. O Deltatrac-II usa a concentração de dióxido de carbono e oxigênio para deduzir seus volumes absolutos. A concentração de dióxido de carbono $\left(\mathrm{FeCO}_{2}\right)$ habilita a dedução do $\mathrm{VCO}_{2}$, e o volume de oxigênio $\left(\mathrm{VO}_{2}\right)$ é calculado com base na diferença das concentrações de oxigênio entre a amostra inspirada e expirada (Sayeed 2003).

As cutias são roedores dasiproctídeos tipicamente neotropicais, com várias espécies distribuídas pelas Américas do Sul e Central, ainda pouco estudadas quanto a seus aspectos biomédicos. O objetivo deste experimento foi aferir a taxa metabólica basal na espécie Dasyprocta azarae, característica da fauna meridional brasileira, e examinar o escalonamento do metabolismo basal nesses roedores, como base para o processo de extrapolação alométrica, que se constitui na melhor ferramenta de auxilio aos clínicos de animais selvagens no cálculo de necessidades nutricionais e de doses de fármacos na terapêutica desses animais.

\section{MATERIAL E MÉTODOS}

Foram utilizadas 34 cutias (Dasyprocta azarae) adultas e sadias, sendo 9 machos não castrados, 9 machos castrados e 16 fêmeas, disponibilizadas pelo Criadouro Científico do Museu de História Natural Capão da Imbuia, do Departamento de Zoológico da Prefeitura Municipal de Curitiba.

Inicialmente todos os animais foram clinicamente avaliados por meio de exame físico, sendo a seguir pesados, marcados e submetidos a punção venosa para colheita de sangue, visando a análise de parâmetros hematológicos (hemograma completo) e bioquímicos (uréia, creatinina e glicemia). A colheita de sangue foi realizada por punção da veia safena lateral, próximo à linha média, na região inguinal, de acordo com a técnica recomendada por Pachaly et al. (2001).

Para a determinação do período ideal de jejum necessário para aferição da taxa de metabolismo basal em Dasyprocta azarae, foi determinada inicialmente a curva glicêmica para animais dessa espécie criados em cativeiro, considerando como glicemia normal valores de $104,5 \pm 33,4 \mathrm{mg} / \mathrm{dL}$ para fêmeas e $130 \pm 13,89 \mathrm{mg} / \mathrm{dL}$ para machos (Pachaly et al. 2001). Foram selecionados aleatoriamente dez animais, que foram alimentados e a seguir submetidos seqüencialmente a colheitas de sangue com intervalos de 5 minutos entre cada animal. $O$ procedimento foi repetido a cada hora, durante doze horas, e todos os indivíduos apresentaram valores normais de glicemia, a partir de cinco horas e até oito horas após a captura, sendo então determinado que seis horas fosse o tempo ideal de jejum.

\section{Determinação da taxa metabólica basal}

Para determinação da taxa metabólica basal os animais foram divididos aleatoriamente em cinco grupos. Empregou-se o monitor metabólico Deltatrac-II ${ }^{6}$, e a aferição dos parâmetros foi executada em dias diferentes para cada grupo, considerando os tempos necessários para jejum e execução do procedimento, e levando ainda em conta que tais animais têm hábitos alimentares diurnos.

Os animais foram capturados em intervalos de 40 minutos, logo após a alimentação, submetidos à aferição da temperatura retal e peso corporal, acondicionados a seguir em caixas individuais de contenção e assim transportados para uma sala com temperatura controlada $\left(22,0 \pm 1,0^{\circ} \mathrm{C}\right)$. A seguir, foram transferidos para caixas plásticas pretas ${ }^{7}$ com volume de 40 litros, nas extremidades de cujas tampas foram acoplados os adaptadores de nylon da máscara que acompanha o monitor metabólico. Um dos adaptadores, no momento da mensuração, era acoplado à mangueira em forma de traquéia que levava o ar expirado ao aparelho, enquanto o segundo adaptador, pelo qual o ar ambiental entrava na caixa, era acoplado à linha de medição da concentração de oxigênio e dióxido de carbono. Os animais permaneciam em repouso durante 40 minutos, dentro das caixas, antes da medição do consumo basal de oxigênio, para adaptação às condições ambientais padronizadas, respeitando intervalo e ordem de captura, e perfazendo um total de seis horas de jejum, até o início da medição.

Antes de iniciar a primeira medição do dia, o monitor metabólico era calibrado com a mistura gasosa própria para tal finalidade, contendo $95 \%$ de oxigênio e $5 \%$ de dióxido de carbono.

\footnotetext{
${ }^{6}$ Deltatrac II, Datex-Ohmeda, Helsinki, Finlândia.

${ }^{7}$ Caixa modelo 1.0/9, Marfinite, Itaquaquecetuba, Brasil.
} 
Após o período de repouso conectava-se a mangueira do monitor metabólico ao adaptador da caixa de medição, iniciando-se a determinação do consumo basal de oxigênio e demais parâmetros necessários para o cálculo da taxa metabólica, por meio de estequiometria.

Cada animal foi monitorizado durante 35 minutos, sendo que os cinco primeiros minutos de cada medição foram descartados pelo monitor metabólico Deltatrac-II, na apresentação da média do dispêndio energético, pois esse tempo é necessário para estabilização do paciente e troca do ar existente nas diversas partes do equipamento e da caixa de medição.

Decorridos os 35 minutos de aferição, eram impressos os relatórios finais, contendo os resultados da medição minuto a minuto com especificação do horário, assim como os valores médios de volume de $\mathrm{CO}_{2}$ produzido ( $\mathrm{ml} /$ minuto), volume de $\mathrm{O}_{2}$ consumido ( $\mathrm{ml} /$ minuto), quociente respiratório e dispêndio energético ( $\mathrm{kcal} / 24$ horas), e a mangueira era desacoplada da caixa de medição, sendo cada animal encaminhado a um ambulatório, para ser submetido a novo exame físico. Após a remoção de cada animal, o equipamento era preparado para ser acoplado à caixa seguinte, contendo o próximo indivíduo a ser submetido à aferição da taxa metabólica basal, respeitando-se o tempo de repouso.

A inferência estatística comparou a taxa metabólica específica (TME) dos indivíduos de cada grupo, facilitando a análise, compreensão e comparação destes dados, pois neste caso não ocorre interferência da variável independente massa corpórea. Os testes estatísticos iniciaram pela determinação das médias e desvios padrão da TME de cada indivíduo, para relacioná-los à massa corporal. A seguir foi realizado o teste de rejeição de pontos experimentais, pelo método de Chauvenet, com o objetivo de descartar possíveis pontos amostrais que pudessem conter desvios grosseiros, evitando-se assim erros que ocorreriam ao considerar tais pontos no tratamento estatístico (Souza 2003).

Para comparação entre as variâncias e as médias de TME dos três grupos, foram realizados testes de hipóteses (comparação entre amostras), conforme descrito por Newbold (1995) e Souza (2003), com a finalidade de constatar se os desvios e as médias desses grupos eram estatisticamente iguais. Foi considerada como hipótese nula $\left(\mathrm{H}_{0}\right)$, em todos os casos, a igualdade entre as médias ou entre as variâncias dos grupos testados. Como hipótese alternativa $\left(\mathrm{H}_{1}\right)$, na comparação entre fêmeas e machos não castrados, foi testada a hipótese da taxa metabólica específica (TME) de machos ser maior que de fêmeas, pois segundo Guyton \& Hall (2002) e Ganong (1989) indivíduos do sexo masculino têm taxas metabólicas maiores que indivíduos do sexo feminino. Como não foram encontrados, na literatura consultada, dados com relação aos machos castrados, foi também testada a hipótese alternativa $\left(\mathrm{H}_{1}\right)$, de seu metabolismo ser diferente do metabolismo dos machos não castrados e também das fêmeas. No caso da comparação entre variâncias, em todos os casos, foi testada a hipótese alternativa das variâncias serem diferentes.

Após estes testes, os dados dos três grupos, e do grupo que pode ser formado considerando os testes de hipóteses, foram submetidos ao teste de normalidade pelo método analítico de Kolmogorov-Smirnov (K-S), pois segundo Souza (2003), os resultados dos testes de hipóteses e as estimativas dos intervalos de confiança só podem ser considerados válidos se os dados experimentais possuírem boa aderência à curva normal. Constatada distribuição normal dos dados, os intervalos de confiança foram aferidos, adotando-se grau de confiança de $95 \%$, sendo esta a probabilidade do respectivo intervalo conter o valor do parâmetro para a população (Souza 2003). Após verificação do intervalo de confiança, foi realizada a análise de regressão linear, pois segundo Withers (1992), a taxa metabólica de animais relaciona-se à massa corporal através da forma geral de uma curva de potência, representada pela equação $\boldsymbol{y}=\boldsymbol{a} \boldsymbol{M}^{\boldsymbol{b}}$, função esta linearizável.

Para observar a tendência de variação conjunta da massa corporal com a taxa metabólica específica (TME) e com a taxa metabólica basal (TMB), foi realizado o teste correlação de pontos experimentais, pois a interpretação dos valores dos coeficientes de correlação $r$ obtidos das amostras fornece uma idéia dos graus e dos sinais das correlações. Após esses procedimentos, foram executados ainda os testes de hipóteses dos coeficientes de correlação, pois segundo Souza (2003), os valores de $r$ são calculados com base nos $\boldsymbol{n}$ elementos das amostras aleatórias, e portanto, representam uma estimativa dos verdadeiros coeficientes de correlação populacionais ñ. Devido a este fato, foram aplicados os testes de hipóteses aos coeficientes de correlação das amostras, conforme preconizado por Souza (2003).

\section{RESULTADOS}

Todos os animais submetidos à aferição da taxa metabólica basal apresentaram parâmetros normais ao exame físico, realizado antes e após o procedimento.

Como os resultados da taxa metabólica basal medida com o monitor metabólico Deltatrac-II são obtidos de forma individual, e os dados de literatura sugerem que hormônios sexuais produzem alterações significativas na taxa metabólica basal, após a aferição em todos os animais optou-se por tabular estes dados nos seguintes grupos:

-Grupo 1, composto por 16 espécimes fêmeas, com massa corporal média de $3.979 \mathrm{~g} \pm 433 \mathrm{~g}$.

Grupo 2, composto por 9 espécimes machos não castrados, com massa corporal média de $3.515 \mathrm{~g} \pm 474 \mathrm{~g}$.

-Grupo 3, composto por 9 espécimes machos castrados, com massa corporal média de $4.546 \mathrm{~g} \pm 304 \mathrm{~g}$.

Os resultados foram também plotados em diagrama de dispersão (Fig.1)

Foram calculados média e desvio padrão da TME (kcal/ $\mathrm{g} / \mathrm{dia}$ ) para cada grupo, obtendo-se para as fêmeas TME média de 0,0552 kcal/g/dia, com desvio padrão de 0,0062 $\mathrm{kcal} / \mathrm{g} / \mathrm{dia}$; para machos não castrados, TME média de $0,0658 \mathrm{kcal} / \mathrm{g} / \mathrm{dia}$ e desvio padrão de 0,0101 kcal/g/dia; e para machos castrados, TME média de $0,0522 \mathrm{kcal} / \mathrm{g} / \mathrm{dia}$, com desvio padrão de $0,0042 \mathrm{kcal} / \mathrm{g} / \mathrm{dia}$.

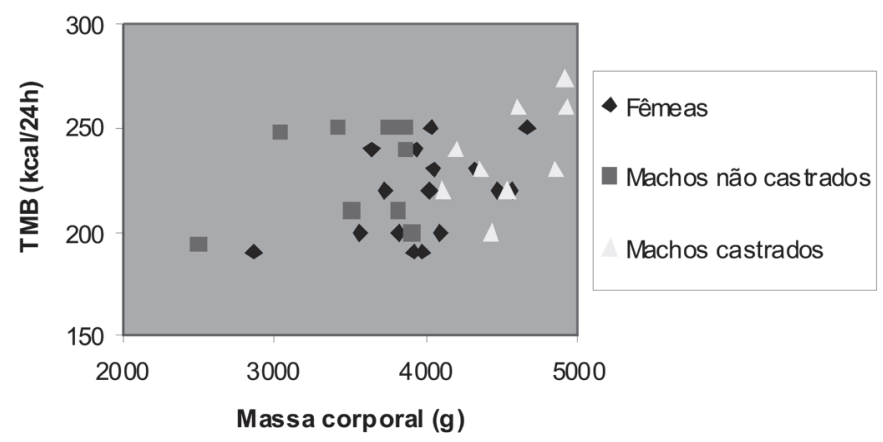

Fig.1. Diagrama de dispersão mostrando a distribuição da TMB (kcal/24h) dos indivíduos em relação à suas massas corporais $(\mathrm{g})$. 
Quadro 1. Intervalos de confiança das médias e variâncias de TME para Dasyprocta azarae, com grau de confiança de $95 \%$, para as populações de fêmeas, machos não castrados e machos castrados, e para a população constituída por fêmeas e machos castrados

\begin{tabular}{lcc}
\hline \multicolumn{1}{c}{ Populações } & \multicolumn{2}{c}{ Intervalos de confiança } \\
\cline { 2 - 3 } & Média $(\mathrm{kcal} / \mathrm{g} / 24 \mathrm{~h})$ & Variância $(\mathrm{kcal} / \mathrm{g} / 24 \mathrm{~h})$ \\
\hline Fêmeas & $0,0519-0,0585$ & $0,00002078-0,00009122$ \\
Machos não castrados & $0,0580-0,0736$ & $0,00004669-0,00037554$ \\
Machos castrados & $0,0489-0,0554$ & $0,00000816-0,00006560$ \\
Fêmeas e Machos castrados & $0,0518-0,0565$ & $0,00001949-0,00002062$
\end{tabular}

O teste de rejeição de pontos experimentais não apontou necessidade de rejeição de nenhum animal, pois todos os valores obtidos ficaram dentro dos limites esperados.

\section{Testes de hipóteses (comparação entre amostras)}

Comparação entre fêmeas e machos não castrados. Com o resultado dos cálculos referentes ao teste de hipóteses para comparação entre as variâncias da TME do grupo de fêmeas e de machos não castrados, aceitou-se a hipótese nula $\left(\mathrm{H}_{0}\right)$ de que as variâncias dos dois grupos são iguais, ao nível de 5\% de significância. Já na comparação entre as médias da TME desses grupos, foi aceita a hipótese alternativa $\left(\mathrm{H}_{1}\right)$ de que a TME do grupo de machos não castrados é maior que a TME do grupo de fêmeas, também ao nível de $5 \%$ de significância.

Comparação entre machos não castrados e machos castrados. Na comparação entre as variâncias da TME dos machos não castrados e dos machos castrados, foi aceita a hipótese nula de que as variâncias destes grupos são iguais, ao nível de 5\% de significância. Entretanto, na comparação das médias de TME destes grupos a hipótese nula foi rejeitada, sendo aceita a hipótese alternativa de que as médias da TME dos dois grupos são diferentes, também ao nível de $5 \%$ de significância.

Comparação entre fêmeas e machos castrados. Comparando as variâncias da TME do grupo de fêmeas e de machos castrados, foi aceita a hipótese nula de que as variâncias são iguais, ao nível de 5\% de significância. Na comparação entre as médias da TME desses dois grupos, a hipótese alternativa foi rejeitada, sendo aceita a hipótese nula de que a TME destes grupos é igual, ao nível de 1\% e 5\% de significância.

\section{Teste de normalidade}

Além dos três grupos iniciais (fêmeas, machos não castrados e machos castrados), foi formado um quarto grupo envolvendo as fêmeas e os machos castrados, para ser submetido ao teste de normalidade, pois o resultado dos testes de hipótese para comparação entre amostras demonstrou que a TME das fêmeas é estatisticamente igual a TME dos machos castrados. Foi aceita a hipótese de normalidade para todos os grupos, ao nível de $5 \%$ de significância $(P>0,05)$.

\section{Intervalo de confiança}

Os intervalos de confiança foram calculados adotandose um grau de confiança de $95 \%$, sendo esta a probabili- dade de que os intervalos de confiança contenham o valor real da taxa metabólica específica (TME) das populações de fêmeas, machos não castrados e machos castrados, além da população que reúne fêmeas e machos castrados, e que foram sendo analisadas em conjunto devido aos resultados dos testes de hipóteses e de normalidade. O Quadro 1 mostra os intervalos de confiança para média e variância da TME das populações em questão.

\section{Análise de regressão}

Houve uma grande variação entre os coeficientes e expoentes de massa estimados para os grupos em estudo (Quadro 2).

Quadro 2. Coeficientes de massa a e expoentes de massa $b$, estimados por regressão linear, para os grupos de fêmeas, machos não castrados e machos castrados, e para o grupo formado com as fêmeas e machos castrados

\begin{tabular}{lcc}
\hline \multicolumn{1}{c}{ Grupos } & $\begin{array}{c}\text { Coeficiente } \\
\text { de massa } \boldsymbol{a} \\
(\mathrm{kcal} / \mathrm{g} / 24 \mathrm{~h})\end{array}$ & $\begin{array}{c}\text { Expoente de } \\
\text { massa } \boldsymbol{b}\end{array}$ \\
\hline Fêmeas & 8,0414 & 0,3979 \\
Machos não castrados & 36,9867 & 0,2224 \\
Machos castrados & 0,1854 & 0,8491 \\
Fêmeas e machos castrados & 3,1526 & 0,5116
\end{tabular}

\section{Correlação de pontos experimentais}

Os grupos de fêmeas, machos não castrados, machos castrados e o grupo que pode ser formado com as fêmeas e os machos castrados apresentaram os seguintes valores de coeficiente de correlação, respectivamente: 0,4649; 0,$2637 ; 0,5919$ e 0,5976 .

\section{DISCUSSÃO}

Segundo Feldman \& McMahon (1983) e Withers (1992), o conhecimento atualmente disponível sobre metabolismo de vertebrados é muito vasto e se baseia, em grande parte, nos trabalhos de Kleiber, realizados nas décadas de 30 e 40, tendo este pesquisador demonstrado que a relação entre a taxa metabólica e a massa corporal não é linear, e proposto o expoente 0,75 para expressar o metabolismo basal com relação à massa corporal em comparações interespecíficas. De acordo com Pachaly \& Brito $(2000,2001)$ e Pachaly (2006), o expoente 0,75 tem sido utilizado para cálculo de taxa metabólica basal de vertebrados, com diversos propósitos, tanto em pesquisa básica quanto aplicada, e que 
a taxa de metabolismo basal é considerada o melhor meio de comparação entre organismos diferentes. Trata-se de uma relação fundamental que existe entre todos os organismos, sendo também a base para o processo de extrapolação alométrica, considerado por estes pesquisadores uma ferramenta capaz de auxiliar os clínicos de animais selvagens, no cálculo de doses das drogas empregadas para a contenção farmacológica ou para o tratamento médico. $\mathrm{McNab}$ (1988) afirma que as relações alométricas são geralmente descritas como uma função exponencial da massa corporal, alegando que a conexão entre um caráter e o tamanho corporal geralmente não é linear.

Segundo Gibbons etal.(1988), Sedgwick (1988a,b), Sedgwick \& Pokras (1988), Sedgwick et al. (1990), Mader (1991), Sedgwick (1991), Fowler (1993), Jacobson (1995), Dorrestein (1997), Gamble et al. (1997), Heard (1997), Pachaly \& Brito $(2000,2001)$ e Pachaly (2006), a fórmula geral para o cálculo da taxa metabólica basal dos animais vertebrados, tanto domésticos como selvagens, é expressa pela equação TMB = a . $\boldsymbol{M}^{\mathbf{0}, 75}$, equação esta contestada por Heusner (1982), que reuniu um conjunto cuidadosamente escolhido de dados da literatura, com o objetivo de reexaminar o escalonamento do metabolismo basal e ao invés de empregar análise de regressão simples, introduziu dois níveis de variação na variável independente: interespecífica e intra-específica e encontrou um expoente de massa de $2 / 3(0,67)$ para o escalonamento intraespecífico. Savage et al. (2004) reexaminando dados, inclusive os utilizados por Heusner (1982), afirmam que, dependendo de quais dados e de como estes são analisados, expoentes entre $2 / 3 \mathrm{e}^{3 / 4}$ podem ser obtidos da TMB de mamíferos, contudo, White \& Seymour (2004) encontraram expoente $0,686 \pm 0,014$ e afirmaram que condições não basais podem influenciar o expoente. Packard \& Birchard (2008) afirmam que White \& Seymour (2004) aparentemente não consideraram desvios de pontos experimentais e que, reanalisando os dados do elefante, este foi um desvio, portanto, o expoente 3/4 não está bem apoiado.

Schmidt-Nielsen (1997), entretanto, também defende o expoente 0,75 , afirmando que as inclinações das retas de regressão estão mais próximas de 0,75 do que de 0,67.

Os resultados do presente estudo, obtidos por análise de regressão linear, conforme preconizado por Withers (1992), diferem dos defendidos por Heusner (1982), Feldman \& McMahon (1983), Gibbons et al. (1988), Sedgwick (1988a,b), Sedgwick \& Pokras (1988), Sedgwick etal. (1990), Mader (1991), Sedgwick (1991), Withers (1992), Fowler (1993), Jacobson (1995), Dorrestein (1997), Gamble et al. (1997), Heard (1997), Schmidt-Nielsen (1997), Pachaly \& Brito $(2000,2001)$ e Pachaly (2006), indicando ser ainda necessário a realização de outros experimentos com uma amplitude maior da massa corporal dos animais em estudo, pois com o aumento da amplitude na variável independente, pode-se ter uma alteração significativa na dispersão da amostra, e conseqüentemente alterações nas inclinações das retas de regressão.

A taxa metabólica específica utilizada em várias etapas da análise estatística deste trabalho foi obtida conforme preconizado por Pachaly \& Brito $(2000,2001)$ e Pachaly
(2006), dividindo-se a taxa metabólica basal pela massa corporal do animal.

Os testes de hipóteses para comparação entre amostras, realizados conforme o descrito por Newbold (1995) e Souza (2003) demonstraram que a média da taxa metabólica específica do grupo de machos não castrados é maior que a do grupo das fêmeas, compatível com a afirmação de Guyton \& Hall (2002), de que o hormônio sexual masculino pode aumentar o metabolismo basal em cerca de $10-15 \%$, enquanto que o hormônio sexual feminino talvez contribua com uma pequena percentagem, e com a afirmação de Ganong (1989) de que a taxa metabólica de machos é maior que a de fêmeas. Tais afirmações são compatíveis também com o resultado dos testes de hipóteses do coeficiente de correlação, que indica haver outra variável afetando a taxa metabólica dos machos não castrados, que não a massa corporal, o que por sua vez é compatível com a menção de Feldman \& McMahon (1983), que esperam que considerações simultâneas de outras variáveis possam resultar em novos achados, mas que as novas variáveis não invalidam as antigas.

Os testes de hipóteses para comparação entre amostras indicaram também que a taxa metabólica média dos machos castrados é estatisticamente igual à das fêmeas, o que sugere que o hormônio sexual masculino referido por Guyton \& Hall (2002) seja a testosterona, porseresteo principal hormônio sexual que sofre depleção com a orquiectomia. Tal resultado é compatível também com a afirmação de Greenstein \& Wood (2006), de que a testosterona eleva a taxa metabólica basal, devido ao aumento da síntese de enzimas e outras proteínas. Contudo, Buttemer \& Astheimer (2000) não encontraram diferença na taxa metabólica de Lichenostomus penicillatus com administração exógena de testosterona.

O teste de normalidade demonstrou que as amostras de todos os grupos seguiram os padrões de distribuição normal, o que segundo Souza (2003), permite utilizar os conceitos de estatística indutiva ou inferência estatística e obter dados da população a partir de dados da amostra. Com isso podemos afirmar que há $95 \%$ de probabilidade de que os intervalos de confiança calculados contenham o valor real da TME de cada grupo estudado, dado este que também não está disponível na literatura consultada.

A afirmação de McNab (1992), de que a maioria dos caracteres de função orgânica, tem uma relação positiva com a massa corporal elevada a uma potência menor que uma unidade, é plenamente compatível com os resultados encontrados, tanto para taxa metabólica basal quanto para taxa metabólica específica, em todos os grupos estudados. Os resultados da taxa metabólica específica confirmam ainda a afirmação de Schmidt-Nielsen (1997), de que o consumo de oxigênio por grama diminui consistentemente com o aumento do tamanho corporal, e com a menção de Withers (1992) ao fato de que um dos mais intrigantes e ainda não resolvidos problemas na fisiologia animal comparativa, é observado na relação entre a taxa metabólica e a massa corporal.

Com o teste de hipóteses para a correlação de pontos experimentais para o grupo de fêmeas e o de machos castrados, constatou-se que há correlação entre a TMB e a massa 
corporal, porém com apenas $10 \%$ de significância, enquanto que para o grupo formado pelas fêmeas e pelos machos castrados a significância foi de $1 \%, 5 \%$ e $10 \%$, podendo indicar que a maior amplitude da massa e o maior $\boldsymbol{n}$ amostral desse grupo, em comparação com os primeiros, pode ter melhorado o coeficiente de correlação.

\section{CONCLUSÕES}

O monitor metabólico Deltatrac-II é um instrumento eficiente para a determinação da taxa metabólica em Dasyprocta azarae, visto que o resultado da aferição da taxa metabólica basal (TMB) de todos os grupos seguiu a distribuição normal, porém não foi possível concluir qual a fórmula ideal para estimar matematicamente a TMB para a espécie em estudo.

A correlação entre TMB e massa corporal foi forte $(1 \%$ de significância) apenas para o grupo formado por fêmeas e por machos castrados, em que a variação na massa corporal foi maior. Já para os grupos compostos apenas por fêmeas ou por machos orquiectomizados, a correlação foi fraca ( $10 \%$ de significância), e para o grupo de machos não castrados não houve correlação. Assim, será necessária a realização de outros experimentos, em que a variação da massa corporal dos animais seja maior e que sejam consideradas também outras variáveis.

O fato de não ter havido correlação entre massa corporal e TMB no grupo de machos não orquiectomizados, aliado à constatação de que a TMB encontrada para este grupo é maior que a encontrada para os grupos de fêmeas e de machos orquiectomizados, que são estatisticamente iguais, indica que os próximos estudos deverão considerar especialmente a variável "hormônio sexual", principalmente a testosterona, na determinação da TMB.

Quanto à avaliação da taxa metabólica específica (TME), os testes de hipóteses para comparação entre amostras permitem concluir que, em Dasyprocta azarae, a TME de fêmeas é igual à de machos orquiectomizados, e que a TME de ambos é inferior à de machos não orquiectomizados.

\section{REFERÊNCIAS}

Buttemer W.A. \& Astheimer L.B. 2000 Testosterone does not affect basal metabolic rate or blood parasite load in captive male white-plumed honeyeaters Lichenostomus penicillatus. J. Avian Biol. 31(4):47988

Dorrestein G.M. 1997. Metabolism, pharmacology and therapy, p.661670. In: Altman R.B., Clubb S.L., Dorrestein G.M. \& Quesenberry K. (Eds), Avian Medicine and Surgery. W.B. Saunders, Philadelphia. 1070p.

Feldman H.A. \& McMahon T.A. 1983. The $3 / 4$ mass exponent for energy metabolism is not an statistical artifact. Resp. Physiol. 52:149-163.

Fowler M.E. 1993. Allometric scaling of nondomestic carnivores for restraint, anesthesia and therapeutics. In: Ibid. (Ed.), Tópicos em Medicina de Animais Selvagens. Curso de Pós-Graduação em Medicina Veterinária, Faculdade de Medicina Veterinária e Zootecnia, USP, São Paulo. 227p. (Apostila)

Gamble K.C., Boothe D.M., Jensen J.M. \& Helmick K.E. 1997. Pharmacokinetics of a single intravenous enrofloxacin dose in scimitar-horned oryx (Oryx dammah). J. Zoo Wildl. Med. 18(4):36-42.

Ganong W.F. 1989. Balanço energético, metabolismo e nutrição, p.236270. In: Ibid. (Ed.), Fisiologia Médica. Atheneu, São Paulo.
Gessaman J.A. \& Nagy K.A. 1988. Energy metabolism: Error in gasexchange conversion factors. Physiol. Zool. 61(6):507-513.

Gibbons G., Pokras M. \& Sedgwick C. 1988. Allometric scaling in veterinary medicine. Aust. Vet. Pract. 18(4):160-164.

Greenstein B. \& Wood D.F. 2006. The Endocrine System at a Glance. $2^{\text {nd }}$ ed. Wiley-Blackwell, Massachusetts. 120p.

Guyton A.C. \& Hall J.E. 2002. Tratado de Fisiologia Médica. 10ª ed. Guanabara Koogan, Rio de Janeiro. 973p.

Heusner A.A. 1982. Energy metabolism and body size. I. Is the 0.75 mass exponent of Kleiber a statistical artifact? Resp. Physiol. 48:1-12.

Heard D.J. 1997. Anesthesia and analgesia, p.807-827. In: Altman R.B., Clubb S.L., Dorrestein G.M. \& Quesenberry K. (Eds), Avian Medicine and Surgery. W.B. Saunders, Philadelphia. 1070p.

Jain N.C. 1993. Essentials of Veterinary Hematology. Lea and Febiger, Philadelphia. $417 \mathrm{p}$.

Jacobson E.R. 1995. Use of antimicrobial therapy in reptiles, p.28-37. In: Ibid. (Ed.), Antimicrobial Therapy in Caged Birds and Exotic Pets. An International Symposium at the North American Veterinary Conference, Orlando.

Mader D.R. 1991. Metabolic scaling of antibiotic dosages, p.632-633. In: Frye F.L. (Ed.), Reptile Care: An atlas of diseases and treatments. Vol.2. T.F.H. Publications, Neptune City. 637p.

McNab B.K. 1988. Complications inherent in scaling the basal rate of metabolism in mammals. Quart. Rev. Biology 63(1):25-54.

Newbold P. 1995. Statistics for Business and Economics. $4^{\text {th }}$ ed. Simon and Schuster, New Jersey. 867p.

Pachaly J.R. \& Brito H.F.V. 2000. Emprego do método de extrapolação alométrica no cálculo de protocolos posológicos para animais selvagens. Hora Vet., Porto Alegre, 20(118):84-90.

Pachaly J.R. \& Brito H.F.V. 2001. Interspecific allometric scaling, p.475-481. In: Fowler M.E. \& Cubas Z.S. (Eds), Biology, Medicine, and Surgery of South American Wild Animals. Iowa State University Press, Ames. $536 p$.

Pachaly J.R., Acco A., Lange R.R., Nogueira T.M.R., Nogueira M.F. \& Ciffoni E.M.G. 2001. Order Rodentia, p.225-237. In: Fowler M.E. \& Cubas Z.S. (Eds), Biology, Medicine, and Surgery of South American Wild Animals. lowa State University Press, Ames. 536p.

Pachaly J.R. 2006. Terapêutica por extrapolação alométrica, p.12151223. In: Cubas Z.S., Silva J.C.R. \& Catão-Dias J.L. (Eds), Tratado de Animais Selvagens: Medicina veterinária. Roca, São Paulo. 1354p.

Packard G.C. \& Birchard G.F. 2008. Traditional allometric analysis fails to provide a valid predictive model for mammalian metabolic rates. J. Exp. Biol. 211:3581-3587.

Savage V.M., Gillooly J.F., Woodruff W.H., West G.B., Allen A.P., Enquist B.J. \& Brown J.H. 2004. The predominance of quarter-power scaling in biology. Funct. Ecol. 18:257-82.

Sayeed A. Validation of Deltatrac II metabolic monitor for neonates. http:/ /www.ee.surrey.ac.uk/Personal/A.Sayeed/deltatrac/Deltatrac.html. Acessado em 1.4.2003.

Schmidt-Nielsen K. 1997. Energy metabolism, p.169-215. In: Ibid. (Ed.), Animal Physiology: Adaptation and environment. $5^{\text {th }}$ ed. Cambridge University Press, Cambridge. 607p.

Sedgwick C.J. 1988a. Finding dietetic needs of captive native wildlife and zoo animals by allometric scaling. Annals American Animal Hospitals Association's 55 $5^{\text {th }}$ Annual Meeting, Washington, p.149150.

Sedgwick C.J. 1988b. Anesthetic and chemical restraint techniques for zoo animals and wildlife. Annals American Animal Hospitals Association's 55 $5^{\text {th }}$ Annual Meeting, Washington, p.162-166.

Sedgwick C.J. 1991. Allometrically scaling the data base for vital sign assessment used in general anesthesia of zoological species. Annals American Association of Zoo Veterinarians Annual Conference, Calgary, p.360-369. 
Sedgwick C.J. \& Pokras M.A. 1988. Extrapolating rational drug doses and treatment periods by allometric scaling. Annals American Animal Hospitals Association's 55 $5^{\text {th }}$ Annual Meeting, Washington, p.156-161.

Sedgwick C.J., Pokras M.A. \& Kaufman G. 1990. Metabolic scaling: Using estimated energy costs to extrapolate drug doses between different species and different individuals of diverse body sizes. Annals American Association of Zoo Veterinarians Annual Conference, South Padre Island, p.249-254.
Souza R.S. 2003. Metodologia de pesquisa e estatística para experimento. Curso de Pós-Graduação em Tecnologias Ambientais, Centro de Ciências Exatas e Tecnológicas, Universidade Federal de Mato Grosso do Sul, Campo Grande, MS. 50p.

White C.R. \& Seymour R.S. 2005. Allometric scaling of mammalian metabolism. J. Exp. Biol. 208:1611-19.

Withers P.C. 1992. Animal energetics, p.82-121. In: Withers P.C. (Ed.), Comparative Animal Physiology. Saunders College Publishing, Fort Worth. 949p. 